\title{
Toxic Epidermal Necrolysis and Epidermolysis Bullosa successfully treated with cultured skin grafts EPIFAST
}

\author{
Massé Sánchez A., Hernández Aguilar G., Mexican Social Security Institute, Pediatric Plastic Surgery, México
}

INTRODUCTION.- Toxic Epidermal Necrolysis (TEN) Most of the cases are drug-induced. Immunological reactions are implicated (keratinocytes apoptosis). Epidermolysis bullosa (EB) is a disease of the skin transmitted genetically is classified (genodermatosis).

OBJETIVE.- To seek an appropriate treatment for patients with Toxic Epidermal Necrolysis (TEN) and Epidermolysis bullosa (EB) since there are no guidelines for it, and the patients behave as burned ones. Therefore, cultured skin grafts "EPIFAST" were used.

METHODOLOGY.-1,2- A 3 and 11 years old female developed an adverse reaction to medication, after $48 \mathrm{hrs,} \mathrm{a} \mathrm{Toxic}$ Epidermal Necrolysis (TEN) was developed in $80 \%$ of the body surface (skin). 3.- 6 years of old male with Convulsive crisis, which makes allergy to Lamotrigina at 7 days, TEN was developed in $75 \%$ of the body surface, meriting intensive care management.

4, 5.- Female 3 and 12 years old with Epidermolysis Bullosa on different parts of the body and infection.

RESULTS.- On the 1st day of treatment, Epifast was applied to 5 patients (50\% of the lesions to patients with NET and $100 \%$ to NB) were removed one week later, and Epifast was applied in the other $50 \%$ of the lesion (NET), the patient with EB healed with a single application of Epifast.

DISSCUSSION.- There are no previous Toxic Epidermal Necrolysis or Epidermolysis bullosa treatments reported in medical literature following these procedures, cultured skin grafts EPIFAST showed to be an ideal proved-treatment for these cases; obtaining a good quality epithelium within 5 days after application.

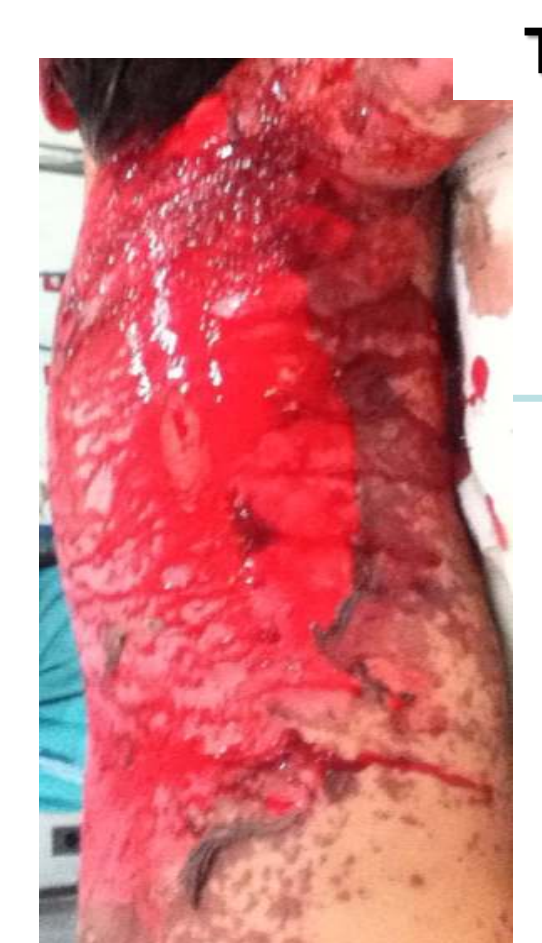

\section{Toxic Epidermal Necrolisis}

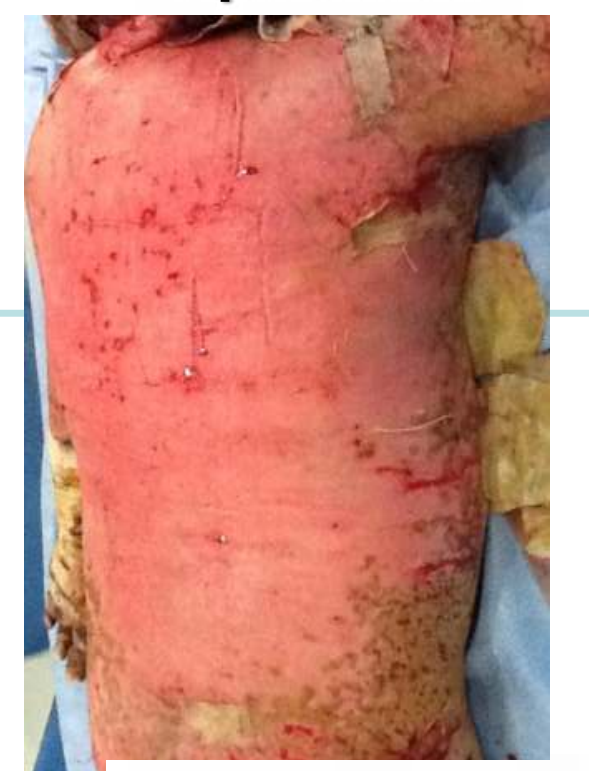

1 and 3 weeks after Epifast
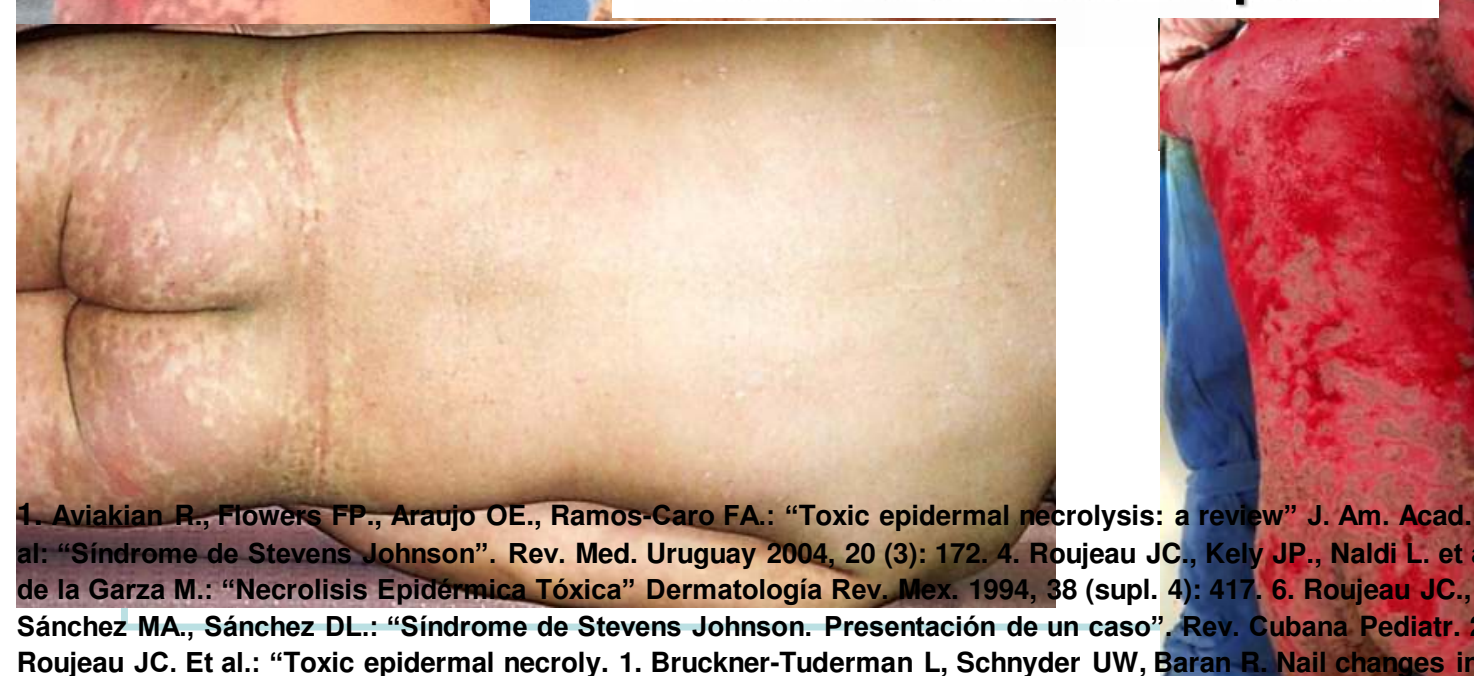

CONCLUSIONS: The surgical technique applied with cultured skin grafts EPIFAST, demonstrated better results than expected, since adequate skin cover throughout the treated surface was achieved. It stimulates the healing and shortened the recovery period of the patient.

\section{Epidermolysis Bullosa}
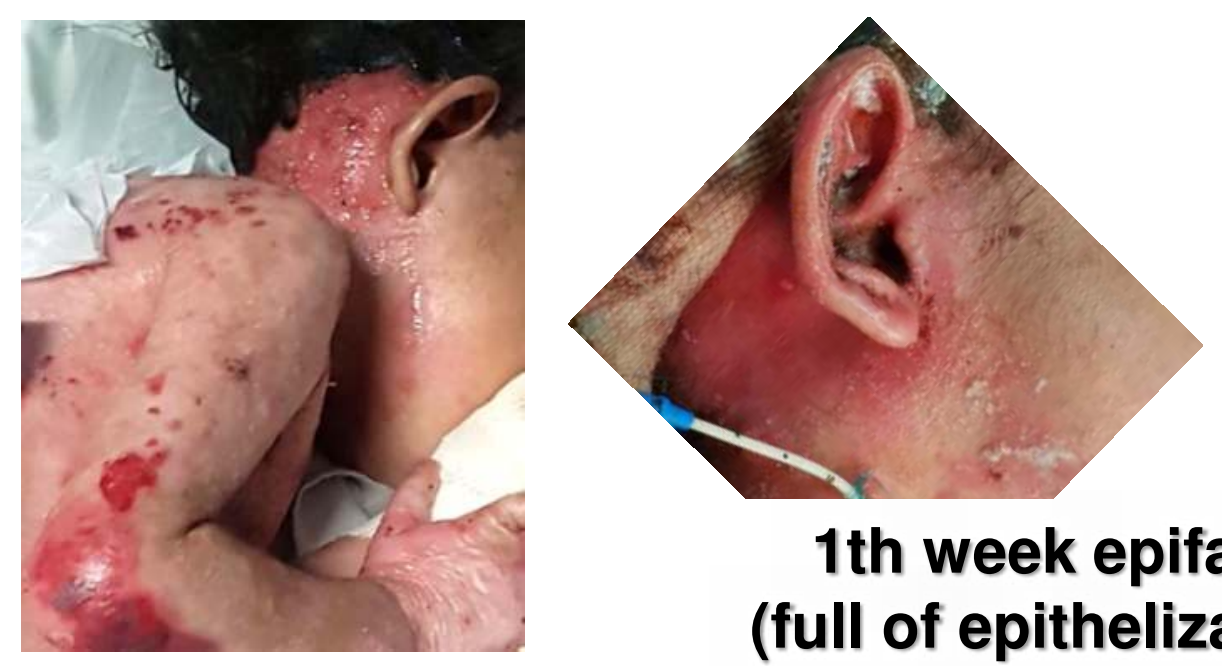

1th week epifast (full of epithelization

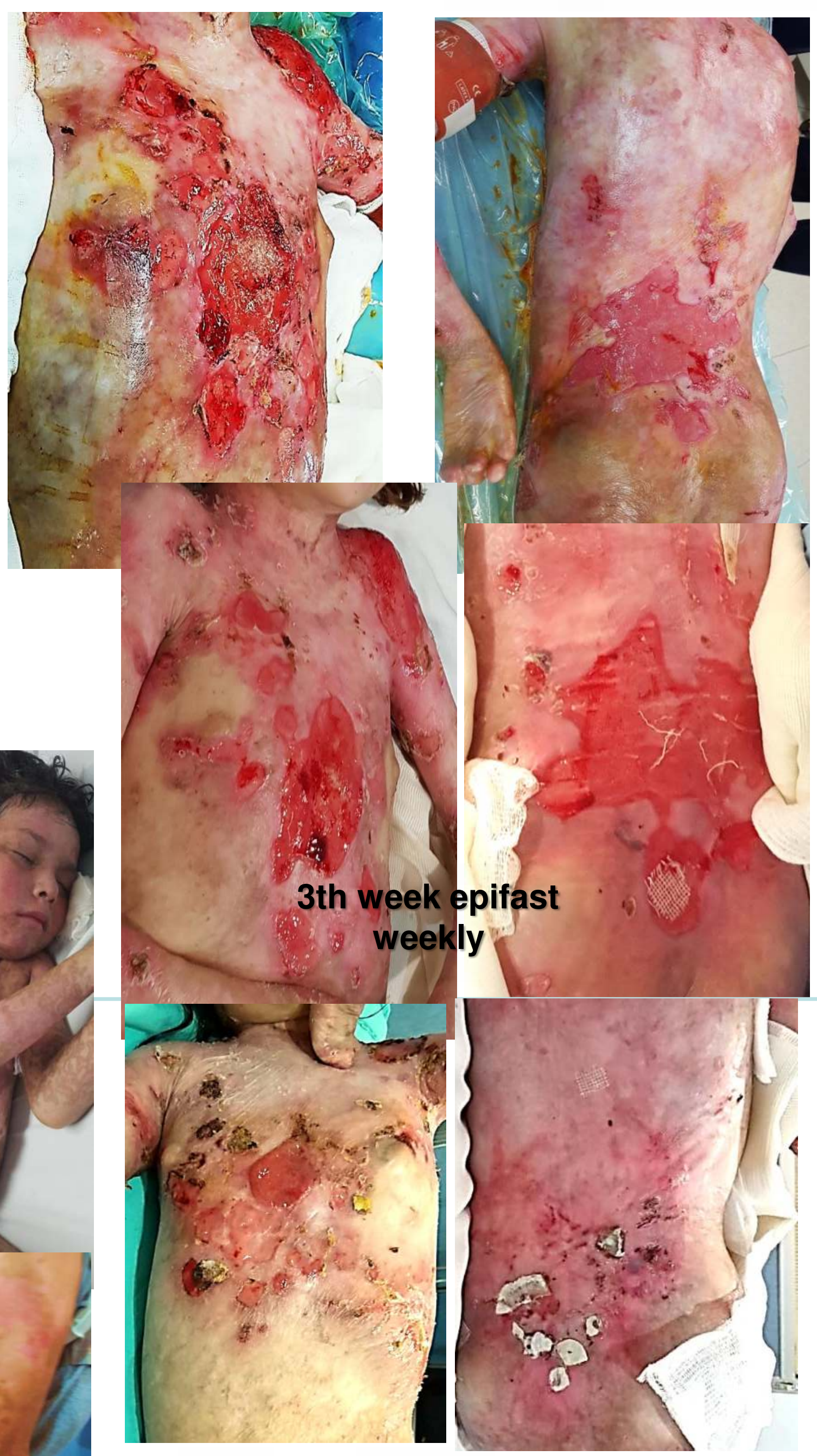

4th Months epifast weekly application, outpatient

25: 69. 2. Roujeau JC., Stern RS.: "Severe adverse culaneous reactions to drugs". New Engl. J. Med. 1994, 331: 1272. 3. Cros A. et on use and the risk of Stevens Johnson Syndrome or toxic epidermal necrolysis", New Engl. J. Med. 1995, 333 (24): 1600. 5. Gómez

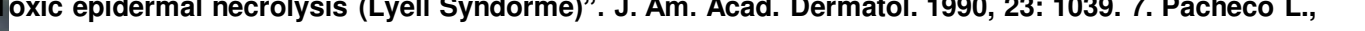

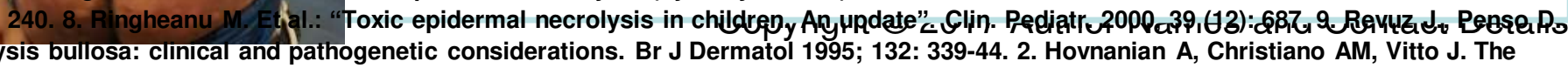

